OPEN ACCESS

Edited by:

Eric Altermann,

AgResearch, New Zealand

Reviewed by:

Peter Mullany,

University College London, UK

Edgardo Sepulveda,

Max Planck Institute for

Developmental Biology, Germany

*Correspondence:

Trudy M. Wassenaar trudy@mmgc.eu

Specialty section:

This article was submitted to Evolutionary and Genomic

Microbiology,

a section of the journal

Frontiers in Microbiology

Received: 21 July 2016 Accepted: 12 September 2016 Published: 27 September 2016

Citation:

Wassenaar TM, Ussery DW and Ingmer H (2016) The qacC Gene Has

Recently Spread between Rolling Circle Plasmids of Staphylococcus,

Indicative of a Novel Gene Transfer

Mechanism. Front. Microbiol. 7:1528.

doi: 10.3389/fmicb.2016.01528

\section{The qacC Gene Has Recently Spread between Rolling Circle Plasmids of Staphylococcus, Indicative of a Novel Gene Transfer Mechanism}

\author{
Trudy M. Wassenaar ${ }^{1 *}$, David W. Ussery ${ }^{2}$ and Hanne Ingmer $^{3}$ \\ ${ }^{1}$ Molecular Microbiology and Genomics Consultants, Zotzenheim, Germany, ${ }^{2}$ Department of Biomedical Informatics, \\ University of Arkansas for Medical Sciences, Little Rock, AR, USA, ${ }^{3}$ Department of Veterinary Disease Biology, Faculty of \\ Health and Medical Sciences, University of Copenhagen, Copenhagen, Denmark
}

Resistance of Staphylococcus species to quaternary ammonium compounds, frequently used as disinfectants and biocides, can be attributed to qac genes. Most qac gene products belong to the Small Multidrug Resistant (SMR) protein family, and are often encoded by rolling-circle $(\mathrm{RC})$ replicating plasmids. Four classes of SMR-type qac gene families have been described in Staphylococcus species: qacC, qacG, qacJ, and $\mathrm{qacH}$. Within their class, these genes are highly conserved, but qacC genes are extremely conserved, although they are found in variable plasmid backgrounds. The lower degree of sequence identity of these plasmids compared to the strict nucleotide conservation of their qacC means that this gene has recently spread. In the absence of insertion sequences or other genetic elements explaining the mobility, we sought for an explanation of mobilization by sequence comparison. Publically available sequences of qac genes, their flanking genes and the replication gene that is invariably present in $\mathrm{RC}$-plasmids were compared to reconstruct the evolutionary history of these plasmids and to explain the recent spread of qacC. Here we propose a new model that explains how qacC is mobilized and transferred to acceptor RC-plasmids without assistance of other genes, by means of its location in between the Double Strand replication Origin (DSO) and the Single-Strand replication Origin (SSO). The proposed mobilization model of this DSO-qacC-SSO element represents a novel mechanism of gene mobilization in RC-plasmids, which has also been employed by other genes, such as InuA (conferring lincomycin resistance). The proposed gene mobility has aided to the wide spread of clinically relevant resistance genes in Staphylococcus populations.

Keywords: gene mobility, qac, SMR, S. aureus, evolution, DNA transfer, rolling circle plasmids

\section{INTRODUCTION}

During investigations of $q a c C$, a gene that provides resistance of Staphylococcus aureus to quaternary ammonium compounds (qac) often used as biocides or disinfectants, we noticed an unusual pattern of sequence conservation amongst various isolates. QacC and related proteins QacG, QacH, and Qac) belong to the protein family of Small MultiResistant proteins (SMR) (Lyon and Skurray, 1987; Wassenaar et al., 2015); they are typically around 100 amino acids long and 
contain two trans-membrane domains. It is assumed they form dimers in the membrane to create a pore through which their substrates are removed from the cell. Some SMR-members of the Qac family are found on long ( $>30,000 \mathrm{bp}$ ) conjugative plasmids, but most are present on small ( $<4000 \mathrm{bp}$ ) plasmids that are maintained by rolling-circle replication. Rolling circle (RC-) plasmids start their replication at a nick-site of a so-called DSO (double-strand replication origin), producing the plus, or leading strand, after which the minus strand is produced from a separate SSO locus (Khan, 2005) (also known as palA element, Gruss et al., 1987). RC-plasmids of Staphylococcus species typically contain only the gene required for initiation of replication, but sometimes a second gene (like $q a c C$ ) is present, and occasionally longer RC-plasmids contain multiple genes.

Studies on the evolutionary relationship of the QacC locus with different plasmids of Staphylococcus spp. date back more than a decade and the mode of spread of this gene remains unexplained. That qacC could be transferred between plasmids had been observed as early as 1995, when it was proposed that the qacC locus of conjugative plasmid pSK41 was the prototype of a so-called qacC cassette (Leelaporn et al., 1995). This view was adopted by others, with the qacC locus of pKS41, which contains a direct repeat flanking qacC, designated as "Type 1" and loci with different flanking regions surrounding qacC classified as "Type 2" and "Type 3" (Bjorland et al., 2001; Alam et al., 2003). The nomenclature was based on a partial sequence of pSK41 that unfortunately had been wrongly assembled. The availability of the complete sequence of pSK41 and closely related plasmids, as well as a large number of sequenced qacC and other qac RC-plasmids, enabled a new comparative analysis. Since most sequence records are available for $q a c C$, this gene was the main focus of the comparison presented here. The nomenclature originally introduced for qacC-bearing plasmids can no longer be maintained. Based on the observed homology patterns amongst different plasmids, we propose a novel mechanism for gene mobilization, which is not exclusive to $q a c C$, as a second resistance gene was identified that most likely also spread using the proposed mechanism.

\section{METHODS}

All plasmid sequences used in this study were downloaded from GenBank. Sequence similarity searches were performed by BlastP and BlastN at NCBI. Multiple alignments were produced by Clustal W or Muscle. Circular homology plots were based on sequence alignments with gaps $>12$ nucleotides removed before calculating the percentage homology using a window of 30 nucleotides. DNA structural properties were assessed as previously described in Pedersen et al. (2000).

\section{RESULTS}

\section{QacC Plasmid Types I and II}

All recorded QacC proteins encoded by RC-plasmids are strictly conserved. Seventy GenBank entries describe identical proteins, sequenced from different staphylococcal plasmids. Only two amino acid (aa) substitutions have thus far been described: an
$\mathrm{A} \rightarrow \mathrm{S}$ substitution at aa position 9 has been recorded five times (four from Staphylococcus warneri plasmids and one from a nonspeciated Staphylococcus spp.) and a change of $\mathrm{I} \rightarrow \mathrm{T}$ at position 30 has been found twice in S. epidermidis plasmids.

Surprisingly, the conservation of qacC at the nucleotide (nt) level is also nearly absolute: in addition to the two mutations resulting in the aa substitutions, only one synonymous mutation has been encountered so far (an $\mathrm{A} \rightarrow \mathrm{G}$ mutation at nt position 81 in pNVH99 of $S$. aureus). Due to this strict conservation, which is indicative of a recent bottle-neck selection event, phylogenetic analysis of the $\mathrm{QacC}$ protein or its gene is not possible, whereas inclusion of the sequences flanking qacC introduces informative variation. Analysis of a number of complete qacC-bearing RCplasmid sequences (selected for their length of approximately $3 \mathrm{~kb}$ ) reveals that these are variable, apart from their strictly conserved qacC gene plus its flanks, as shown in Figure $\mathbf{1}$.

The plasmids used in Figure 1 fall into two types depending on their rep gene, which we define as Type I and Type II, respectively, as summarized in Table 1. Rep1, the determinant for Type I plasmids, is typically 282-307 aa long while Rep2, indicative of Type II, is 332-334 aa. Rep2 homologs were previously described as RepNVH99 (Bjorland et al., 2001) and REP416 (Alam et al., 2003). Both types of plasmids mostly lack other recognized genes and their rep is found invariably upstream of $q a c C$ on the opposite strand, so that the two genes are divergently transcribed.

Even within their types, Rep 1 and Rep2 are still less conserved than QacC: Rep1 is conserved for $77 \%$ at the aa level $(68 \%$ at nt level), and for Rep2 these percentages are 82 and 78\%, respectively. The difference in nt conservation between rep and qacC dictates these genes did not co-evolve on the RC-plasmids where they now reside. Instead, the observation can only be explained by assuming that qacC was recently incorporated into plasmids with variable rep genes, an event that occurred so recently that third-base variation has not yet been introduced.

In an attempt to explain the finding of a conserved qacC in these variable backgrounds we compared the flanking sequences of qacC in the RC-plasmids listed in Table 1. Key features of the multiple DNA alignment are summarized in Figure 2.

Upstream of qacC 162 nucleotides are identical in Type I and Type II plasmids except for two regions: a variable number of direct repeats (DR1) is present toward the distal end of the upstream flank, and an $8 \mathrm{nt}$ repeat insert is found in two Type II plasmids, in a region where a $q a c C^{\prime}$ insert is found in a number of other plasmids, which will be revisited in the next section. The distal end of this 162-long upstream region starts with the DSO, the nature of which will be discussed at the end of this paper. Beyond the DSO, moving further away from qacC in the upstream direction (toward the start of the replication gene), the sequences diverge between Types I and II (Figure 2). Downstream of qacC, Type I and Type II plasmids share $164 \mathrm{nt}$ before their sequences diverge. The exception is pSH416, which contains a large insert, although it cannot be excluded that this is the result of an assembly error.

Thus, the borders of a conserved qacC locus shared between Types I and II plasmids is defined from $162 \mathrm{nt}$ upstream (bearing a DSO element) to $164 \mathrm{nt}$ downstream of the qacC open reading 


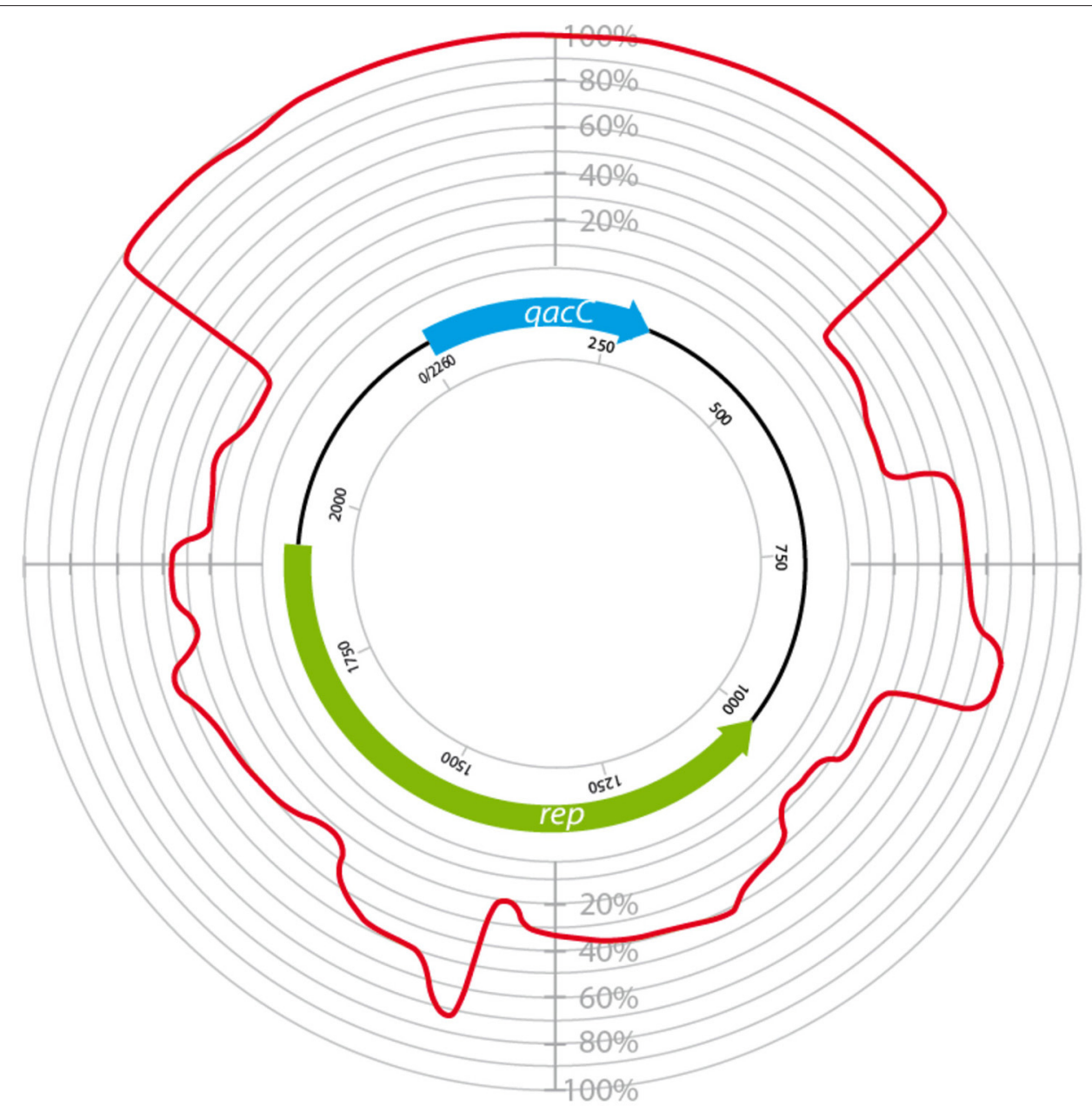

FIGURE 1 | Circular homology plot based on the nucleotide alignment between QacC plasmids. The figure is based on an alignment after gap removal of pNVH99, pWBG754, pSA1308, pLUH01, pKH8, pSH4126, pPI-2, pSW49, pSK89, pWBG32, pSepCH, and pSM52, which were reopened for alignment at the start of qacC. The indicated length of rep is the average of the analyzed genes.

frame, and includes an SSO element. We proposed this "qacC element" has recently been spread over short RC-plasmids.

\section{Plasmids with a Truncated qacC' in Front of a Functional qacC}

As has been previously described, a number of short RC-plasmids contain a repeat of $142 \mathrm{nt}$, composed of the first 97 coding nt of qacC, together with 45 upstream nt (indicated by a gray box in Figure 2). The fragmented open reading frame is frequently annotated as qacC' (previously called Delta-qacC) but the gene fragment is too short to produce a functionally active protein (Leelaporn et al., 1995). Phenotypic evidence from pSM52 (Costa et al., 2013) or pSW49 (Bjorland et al., 2006) suggests that the promoter of $q a c C$ is present on the 45 nucleotides that separate the qacC' fragment from the active, complete gene (the promoter is also present in front of $q a c C^{\prime}$ ).

Plasmids containing the 142-nt duplication of qacC' are designated Type Ia or Type IIa, depending on their Rep (Table 1). That this partial duplication can be variably present on both types can be explained in two ways. Either the qacC locus was distributed to Type I and Type II plasmids first, after which an identical qacC' duplication occurred independently in both, or the duplication occurred first and the locus with or without the duplication spread independently in both types of plasmids. Analysis of the downstream sequences provides a clue as to which scenario is more likely. A single-nucleotide insertion is found $137 \mathrm{nt}$ downstream of qacC in all known Type Ia and Type IIa loci (indicated by an asterisk in Figure 2), and it is also present in some Type II loci (e.g., pSA1308 or pLUH01, see Table 1). It is therefore proposed that the duplication occurred in such a Type II plasmid (Figure 3), after which the whole locus including the duplication was distributed over Type I plasmids. This observation supports the hypothesis that the QacC locus is transferable.

A few Type Ia plasmids contain multiple rep (pseudo)genes, for example pSW49 of S. warneri (Bjorland et al., 2006). This plasmid of 3552 bp length contains, besides qacC', qacC, and rep1, a truncated rep2 pseudogene. Although occasionally RC-plasmids are encountered that contain two different but active replication proteins (e.g., pMRI5.2 of Lactobacillus 
TABLE 1 | Plasmids containing QacC used in the analysis.

\begin{tabular}{|c|c|c|c|c|c|c|}
\hline Type & Characteristic & Plasmid name & $\begin{array}{l}\text { Accession } \\
\text { number }\end{array}$ & $\begin{array}{l}\text { Plasmid } \\
\text { length (bp) }\end{array}$ & Isolated from & Remarks \\
\hline $\begin{array}{l}\text { Short RC- } \\
\text { plasmids }\end{array}$ & & & & & Examples & \\
\hline \multirow[t]{2}{*}{ Type I } & QacC and Rep1 & pSepCH & AY092027 & 2391 & S. epidermidis & \\
\hline & & pSK89 & M37889 & 2390 & S. aureus & \\
\hline \multirow[t]{4}{*}{ Type la } & QacC', QacC, and Rep1 & $\mathrm{pPI}-2$ & AB125342 & 2779 & S. warneri & Extra G 137 bp downstream of QacC \\
\hline & & pSM52 & JX898993 & 2782 & S. aureus & Extra G 137 bp ds downstream QacC \\
\hline & & pSW49 & AM040730 & 3552 & S. warneri & $\begin{array}{l}\text { A9S mutation. Extra G } 137 \text { bp downstream of QacC, } \\
\text { contains Rep2 fragment }\end{array}$ \\
\hline & & pSW174 & AM040729 & 5767 & S. warneri & $\begin{array}{l}\text { A9S mutation. Extra G } 137 \text { bp downstream of QacC, also } \\
\text { contains REP2 }\end{array}$ \\
\hline & & pNVH99 & AJ296103 & 2239 & S. aureus & Silent mutation in QacC \\
\hline & & pLUH01 & FR714928 & 2241 & S. aureus & Extra G 137 bp downstream of QacC. QacC not annotated \\
\hline & & "pSH416" & AY121857 & 2425 & S. aureus & $\begin{array}{l}\text { Unnamed plasmid from strain SH416. Incomplete plasmid } \\
\text { seq. Extra G } 137 \text { bp downstream of QacC }\end{array}$ \\
\hline \multirow[t]{2}{*}{ Type lla } & QacC', QacC, and Rep2 & pSK108 & GQ900464 & 2418 & S. epidermidis & Extra G 137 bp downstream of QacC \\
\hline & & $\mathrm{pKH8}$ & U50077 & 2417 & S. aureus & Extra G 137 bp downstream of QacC \\
\hline \multirow[t]{2}{*}{ Type III } & Direct repeats flank QacC & pST827 & Z37964 & 2831 & Staphylococcus sp. & Isolate described as "meat-associated." Contains Rep2 \\
\hline & & pSP187 & AM040731 & 5550 & S. pasteuri & Contains a different Rep gene plus other genes \\
\hline
\end{tabular}

Long conjugative plasmids

Examples

PSAP082A

pGO1

pSK41

PLW043

PSAP079A

PSAP069A

$\begin{array}{lll}\text { GQ900381 } & 43,807 & \text { S. epidermidis } \\ \text { GQ900434 } & 44,116 & \text { S. aureus } \\ \text { FM207042 } & 54,000 & \text { S. aureus } \\ \text { AF051917 } & 46,445 & \text { S. aureus } \\ \text { AE017171 } & 57,889 & \text { S. aureus } \\ \text { GQ900432 } & 47,322 & \text { S. aureus } \\ \text { GQ900422 } & 42,198 & \text { S. aureus }\end{array}$

GQ900381 43,807
Contains 3 direct repeat units at start of upstream DR1 unit DR3 unit downstream of QacC is truncated

Unclassified

QacC

"pSH651"

AY121858 1545

S. aureus

Unnamed plasmid from strain SH651. IS431 directly downstream of QacC. Incomplete plasmid sequence

Excluded are QacC sequences from GenBank entries that contain insufficient flanking sequences, double entries, or contigs from genomic sequences that were not (yet) fully assembled.

plantarum, Cho et al., 2013), in most cases only one functional replication initiation protein is present. Plasmid pSW49 is possibly a fusion product of a Type Ia and a Type IIa plasmid, in which only one copy of the qacC'-qacC locus was maintained, while Rep2 was truncated. Likewise, S. warneri plasmid pSW174 is likely a fusion plasmid that in this case retained both Rep genes.

Alam et al. (2003) defined a "Type 3" class based on the plasmid of $S$. aureus strain SH651. This unnamed and incompletely sequenced plasmid (called pSH651 in Table 1) contains an insertion sequence directly downstream of qacC. It further contains a $122 \mathrm{nt}$ direct repeat upstream of qacC. The total length of this plasmid is not recorded and further sequences have not been made available, so the exact nature of this plasmid cannot be determined at this stage. However, the $122 \mathrm{nt}$ repeat is part of a sequence that is also repeated in other RC-plasmids bearing qacC, which we call Type III.

\section{Type III qacC Plasmids, Progeny or Precursor?}

The prototype of Type III plasmids is pST827 (Table 1), sequenced from a strain of unspecified "Staphylococcus sp." isolated from food (Heir et al., 1995) (an incorrectly assembled version of this plasmid appeared in Alam et al., 2003). In Type III plasmids, qacC is flanked by two identical 229 nt-long sequences 


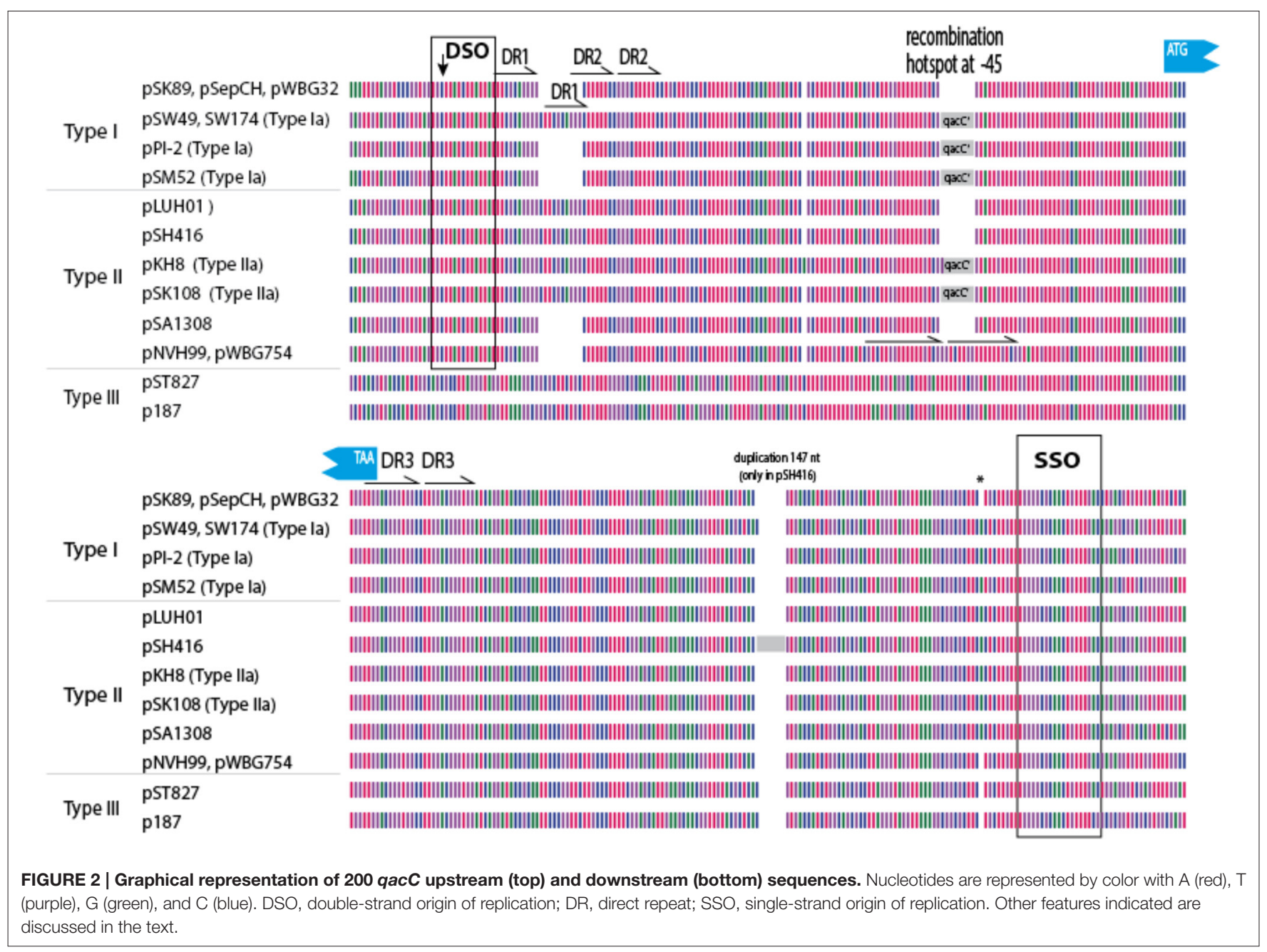

so that the upstream and downstream flanks of qacC are identical, except for one mismatch (an extra T present in the downstream unit). For this reason, Type III plasmids do not match the conservation of the upstream qacC flank (Figure 2) and were excluded from the homology plot in Figure 1.

Two models can be proposed how the qac locus of Type III plasmids relates to Types I and II, shown in Figure 4. The simplest model (Figure 4A) proposes that Type III is formed from a Type I or II qacC plasmid by duplication of the downstream flank inserted into the upstream flank, at position -45 nt. Alternatively (Figure 4B), Types I and II are formed from Type III, which requires a hypothesized precursor in which upstream sequences have been duplicated into the downstream flank. Although that model requires two steps, it may be the more likely scenario, because in the hypothesized precursor a DSO and SSO would be separated by $316 \mathrm{nt}$, a distance that is more typical for staphylococcal RC-plasmids that only contain a rep gene (e.g., pSK6 of S. aureus, acc. nr. NC_001995).

Table 1 lists pSP187 from Staphylococcus pasteuri as a second example of a Type III RC-plasmid. It contains a different replication gene as well as a few other genes-it may have resulted from a recombination with another plasmid. So far, pST827 and
pSP187 are the only completely sequenced short RC-plasmids containing a Type III qacC.

The qac locus of Type III RC-plasmids can also be found on long conjugative plasmids; seven of these (Table 1) were used for comparative analysis (a graphical representation is given in Figure S1). In these plasmids two transposase genes that are part of Insertion Sequence IS257 copies border the locus. In between these ISs a rep1 gene is present. This suggests that a complete Type I plasmid was incorporated into a conjugative plasmid, after which recombination events duplicated the SSO-bearing fragment and deleted the DSO flank immobilized the plasmid. The public database does not contain short qacC RC-plasmids of Type III that contain rep1.

\section{A Hotspot of Recombination Upstream of qacC}

We noted that the insertion of the duplication observed in Type III plasmids occurred at the same position as the insertion of $q a c C^{\prime}$ in Types Ia/IIa. This suggests the -45 position is frequently subject to recombination. Indeed, in two Type II plasmids (pWBG754 and pNVH99), $18 \mathrm{nt}$ are repeated here, replacing 10 nt of original sequence, thus resulting in an insertion of $8 \mathrm{nt}$ in 
the alignment (Figure 2). That the position $45 \mathrm{nt}$ upstream of qacC is a frequent target for recombination may be due to the local physical properties of the sequence. An analysis of the DNA structural properties revealed this is an AT-rich region that is curved and will easily melt.

\section{Other Qac Members of the SMR Family}

Relatively few sequences are available for Qac members of the SMR family other than QacC. Less than 10 records each are currently available for QacG, QacJ, or QacH, and a number of these are contigs from genome or metagenomic sequencing projects. All these plasmids contain a rep2 homolog.

The nucleotide sequences of five QacG plasmids (four RCplasmids and one segment of a conjugative plasmid) listed in Table 2 produce a similar conservation pattern as observed for QacC plasmids: the open reading frame of $q a c G$ is $99 \%$ conserved (5 mismatches only) whereas rep 2 is only $76 \%$ conserved. Again,

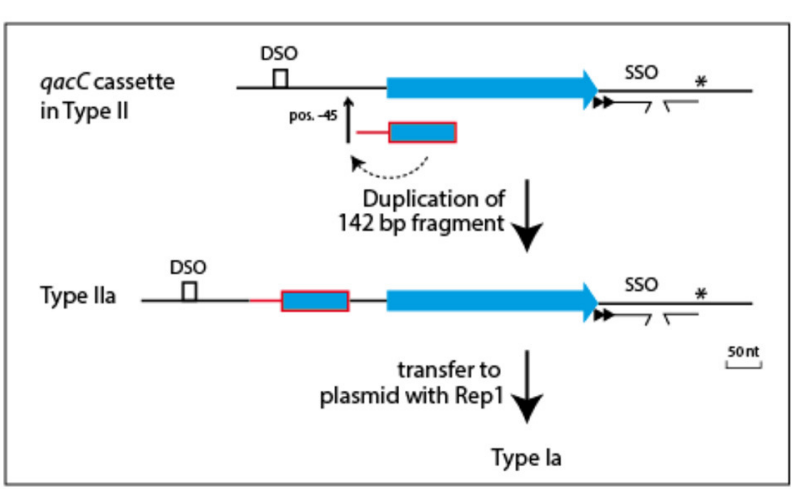

FIGURE 3 | Model for formation of the qacC' fragment present in Types la and Ila plasmids. A single $\mathrm{nt}$ insertion $137 \mathrm{nt}$ downstream of qacC (indicated by the asterisk) is present in some Type II plasmids (e.g., pLUH01) and conserved in both Types la and Ila. This is evidence that the duplication of the 142 nt-long qacC' fragment, inserted at position -45, occurred in a Type II plasmid to form Type Ila, after which the complete locus was mobilized to give Type la. the nearly complete conservation of $q a c G$ is extended to its flanking sequences, encompassing the DSO and SSO, although the homology is continued $22 \mathrm{nt}$ upstream of the DSO nick site. In contrast, the five analyzed QacJ sequences (three from contigs) are stronger conserved for rep2 (91\%) than for qacJ (83\%) and the homology of the rest of the plasmids suggests they are closely related (data not shown). These observations show that there is no functional selection that would prevent SMR-type qac genes from accumulating nt mutations: qacG seems to have been around long enough to collect a limited number of mutations already, in contrast to qacC. There are not enough completely sequenced qacH plasmids publically available to assess their homology.

The homology between plasmids bearing qacC (Type II), $q a c G$, qacJ, and $q a c H$ was used to identify conserved regions (Figure 5). These were restricted to the DSO, a short region immediately downstream of $q a c$, and the distal end of the SSO region.

\section{Conserved DSO and SSO in Three Classes of qac Genes}

Adding sequences of Type I QacC plasmids to the alignment on which Figure 5 was based identified conserved sequences essential for replication, summarized in Figure 6. The distal end of the 165 nt-long QacC upstream flank including the DSO is strictly conserved, though the third base is $\mathrm{G}$ instead of $\mathrm{T}$ in Type I QacC plasmids only. The direct repeat unit DR1 of QacC plasmids is also present in all QacG plasmids, two QacJ plasmids and in the single QacH plasmid that was analyzed. Besides in QacC plasmids, the DR2 unit is repeated in two QacJ plasmids (the three other QacJ sequences contain an alternative DR2). In all plasmids, the DSO is followed by a stretch of low complexity sequences containing multiple short homo-nucleotide repeats. The rest of the upstream flank is not conserved between these Qac plasmids.

The downstream flank of all qac genes is more strongly conserved than the upstream flank. It starts with a $12 \mathrm{bp}$-long direct repeat, (AATTGCTTTATT, indicated as DR3 in Figure 6),

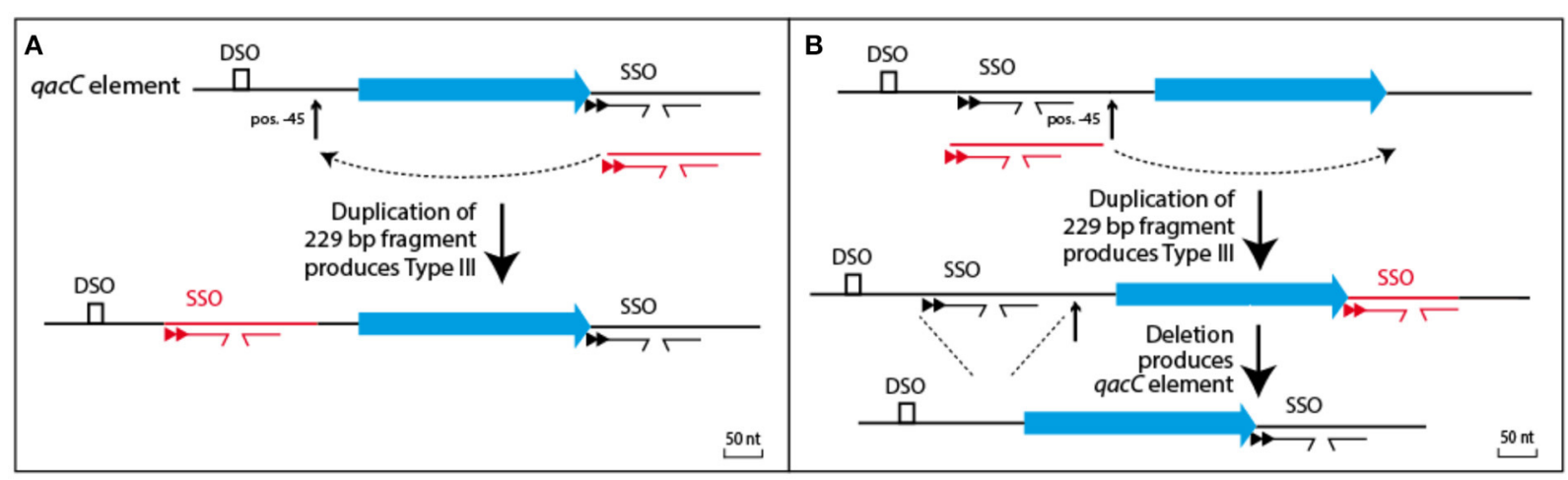

FIGURE 4 | Relationship between Types I/II and Type III plasmids. (A) Type III could be formed from the qacC element by a single duplication event whereby the downstream flank is inserted into a hotspot for recombination, positioned $45 \mathrm{nt}$ upstream of qacC. (B) Alternatively, a precursor can be hypothesized with qacC next to DSO and SSO. A duplication of the SSO-bearing segment would result in Type III, after which a deletion would produce the qacC element. 
TABLE 2 | Plasmids containing QacG, QacJ, or QacH used in the analysis.

\begin{tabular}{|c|c|c|c|c|c|}
\hline $\begin{array}{l}\text { Gene (protein } \\
\text { accession nr) }\end{array}$ & Plasmid name & Accession number & $\begin{array}{l}\text { Plasmid } \\
\text { length (bp) }\end{array}$ & Isolated from & Remarks \\
\hline QacG (087866) & pST94 & Y16944 & 2267 & S. aureus & \\
\hline QacG (YP_006938120) & pSA052A & NC_013332 & 32,445 & S. aureus & Conjugative plasmid, contains truncated Rep2 \\
\hline QacG (WP_002477903) & $\begin{array}{l}\text { "BVSO" from } \\
\text { strain BVS058A4 }\end{array}$ & AGZV01000050 & 2263 & S. epidermidis & $\begin{array}{l}\text { S32T. Rep is annotated as "hypothetical" and } \\
\text { probable assembly error (duplication) at the } \\
\text { circularization border was corrected }\end{array}$ \\
\hline QacG (ALS73700) & pRIVM1076 & CP_013624 & 2216 & S. aureus & \\
\hline QacG (WP_015740450) & pW33578 & NZ_kk038130 & 2538 & S. aureus & Contig \\
\hline QacJ (EQM91153) & pS1d & AUPS01000033 & 2649 & S. aureus & Contig \\
\hline QacJ (CAD55144) & pNVH01 & NC_004562 & 2650 & S. aureus & \\
\hline QacJ (AAB47993) & $\mathrm{pKH} 4$ & U81980 & 2487 & S. aureus & \\
\hline QacJ (WP_052996450) & pNA32 & NZ_CTWR01000064 & 2639 & S. aureus & Contig \\
\hline QacJ (WP_052996449) & p92271 & NZ_CUFY01000074 & 2613 & S. haemolyticu & Contig \\
\hline QacH (CAA76544) & pST2H6 & Y16945 & 2378 & S. saprophyticus & Incomplete plasmid sequence \\
\hline QacH (AAO83011) & pTEF1 & AE016833 & 66,320 & E. faecalis & Not included in the analysis \\
\hline QacH (ACC78797) & - & EU622634 & 225 & S. haemolyticus & Partial open reading frame only \\
\hline
\end{tabular}

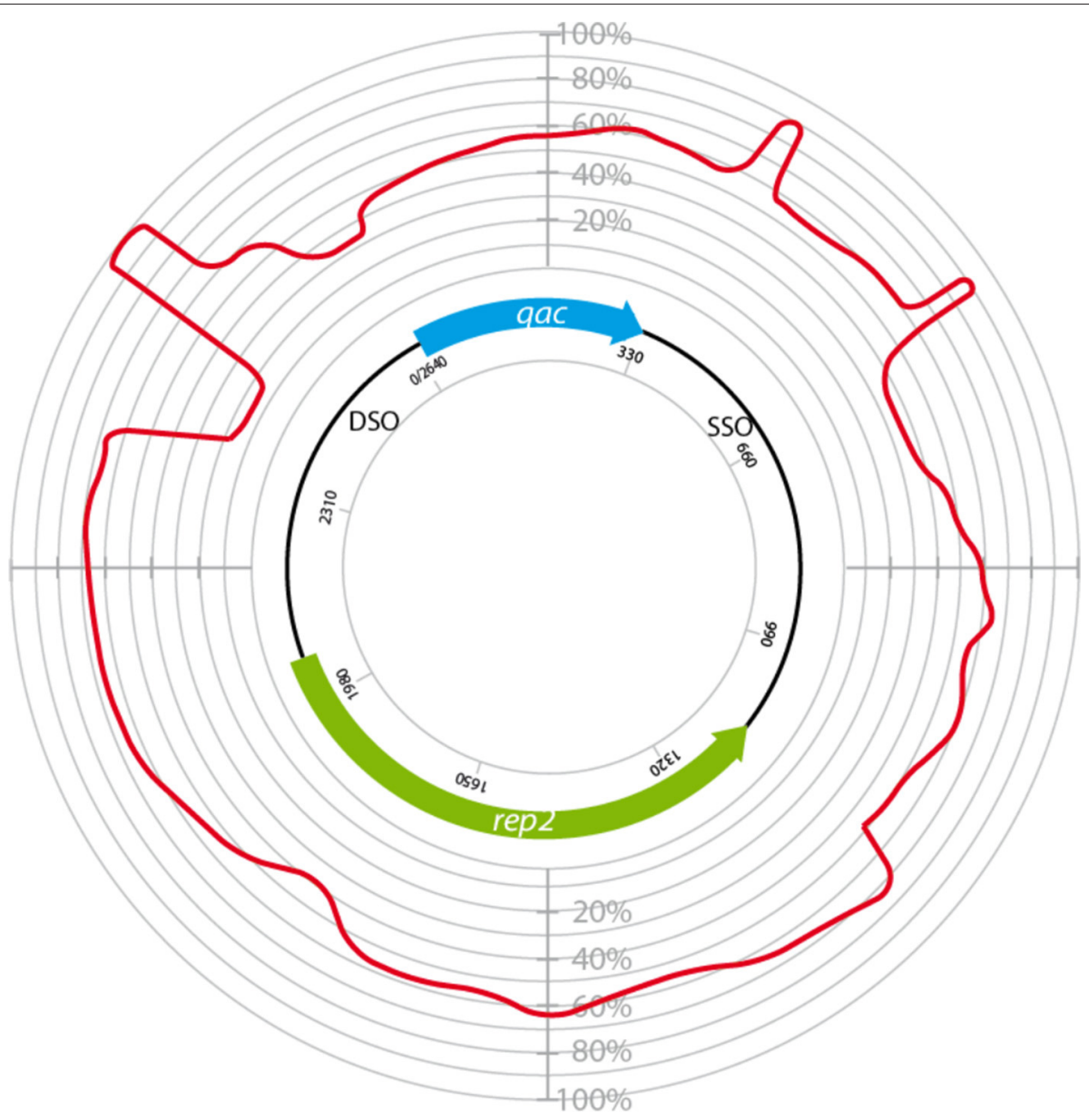

FIGURE 5 | Circular homology plot of plasmids bearing variable qac genes. The homology is based on 4 QacC plasmids (Type II, Table 1) and 5 QacG, 5 QacJ, and 1 QacH plasmids, listed in Table 2. 
A

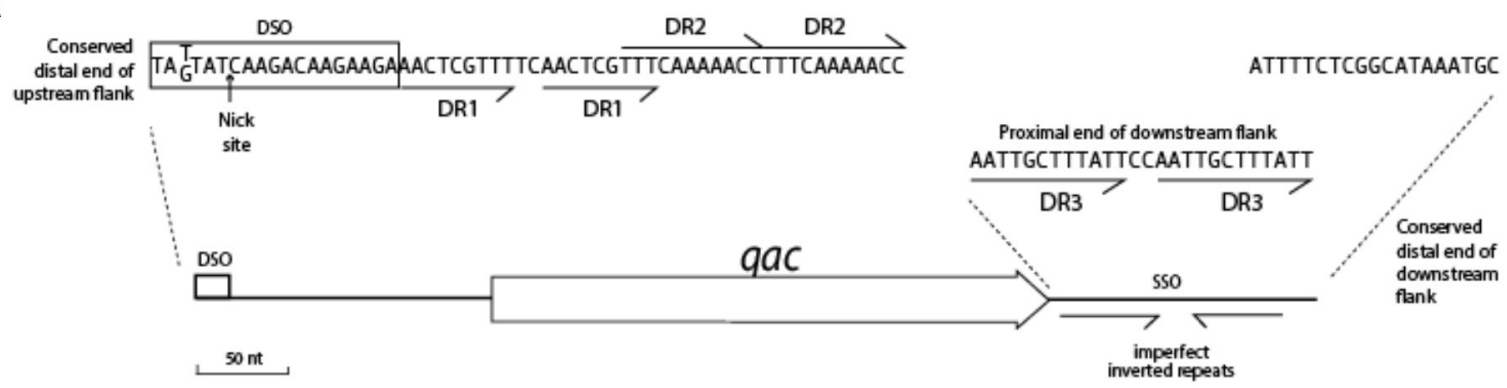

B

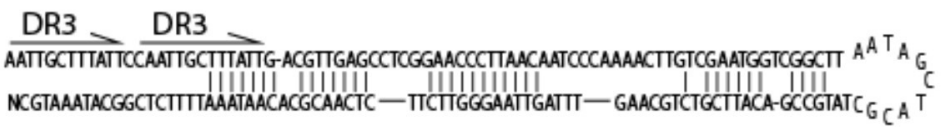

FIGURE 6 | Conserved features of the Qac locus. (A) Sequences indicated are highly conserved in the flanks of 25 qacC, qacG, and qacJ genes. (B) Hairpin structure of SSO for QacC plasmids.

which is completely conserved in case of qacC and qacj; the first unit is mutated in $q a c G$ and both units contain mutations in qacH sequences. The very distal end of the downstream flank contains $18 \mathrm{nt}$ that are strongly conserved ( $q a c J$ contains one mismatch in the last T). Beyond this, homology is lost. Weakly conserved inverted repeats are present in the downstream flank, which can form a hairpin structure (Figure 6B) related to the function of SSO.

The conserved sequence of the DSO given in the box of Figure 6 is the strand on which the qac gene is found. The arrow indicates the position where Rep introduces a nick in the complementary strand, which initiates replication of the plus strand (Gruss et al., 1987; Khan, 2005; Bikard et al., 2010). RC-plasmids are divided into different families depending on the type of replication protein and the DSO sequence this protein recognizes. Originally, four eubacterial RC-plasmid families were recognized (del Solar et al., 1998) which were extended to over 12 (Khan, 2005) and now 17 (Ruiz-Masó et al., 2015) plasmid families. Based on aa similarity, Rep1 of Type I plasmids belongs to the pC194-family (Seery et al., 1993), a representative of the incB incompatibility group (Thomas et al., 1990). The conserved DSO resembles that of pC194 with only one mismatch and one insertion (the DSO of pC194 is TATTAT $\downarrow$ CAAGATAAGAAAGA, Bikard et al., 2010). The nature of Rep2 is less clear, though it is more closely related to Rep of pC194 than to Rep proteins of the other families; information on its inc group could not be identified. Rep2 recognizes the same DSO as Rep1 with the exception of the polymeric nucleotide positioned four bases upstream of the nick site, which is $G$ instead of $\mathrm{T}$.

In a number of well-studied RC-plasmids, the nick site is positioned in the loop of a stem-loop structure that is formed by inverted (palindromic) repeats (Ruiz-Masó et al., 2015). However, from the DSO and sequences surrounding the nick site in Qac plasmids inverted repeats are absent. Lack of hairpin structures in a DSO has been observed before in other members of the pC194-family, for instance in pUB110 of Bacillus subtilis (Alonso et al., 1988). A functional DSO further depends on a binding element for the Rep dimer. This binding element is usually less conserved than the nick site and in case of the pC194 family typically contains inverted repeats (del Solar et al., 1998; Bikard et al., 2010). Since these are absent in case of Qac, we propose that two sets of direct repeats following the nick site function as the binding region for Rep1 and Rep2.

The conserved SSO found in the downstream flank of qacC belongs to the ssoA-type (Kramer et al., 1998). Irrespective of whether Qac is present or absent, this particular SSO sequence is almost exclusively found in Staphylococcus plasmids, in accordance to experimental evidence that SSO sequences are host-specific (Kramer et al., 1998), although promiscuous within the genus.

In the Type III QacC plasmid pST187, where the SSO flank is duplicated, the event has replaced the DSO. Lack of a recognizable DSO element has been pointed out before (Bjorland et al., 2007). These authors proposed an alternative DSO element, and in combination with its unique Rep gene it was suggested that pSP187 belongs to RRC-group VI plasmids (Bjorland et al., 2007).

\section{A Model of Qac Mobility}

The fact that Qac is positioned in between SSO and DSO on RCplasmids may explain how it is mobilized. During rolling circle replication, the plus strand is formed from the nick site, with the replication fork moving all the way round toward the SSO, to continue beyond the original nick site. As Khan describes: "Once the replication fork reaches the termination site, i.e., the regenerated DSO, DNA synthesis proceeds to approximately 10 nucleotides beyond the nick site. (...) The SSO [is] generally located immediately upstream of the DSO such that [it is] not exposed in a single-strand form until the leading strand has been almost fully synthesized" (Khan, 2005). Initiation of replication is tightly regulated, and how re-initiation is inhibited until the 
cycle is completed is well understood for some RC-plasmid families (e.g., pT181, reviewed in Ruiz-Masó et al., 2015), but for the pC194 family, to which Qac RC-plasmids belong, the regulation is not clear (Ruiz-Masó et al., 2015). The presence of a gene $(q a c C)$ in between SSO and DSO is an anomaly for these plasmids, as the sso is usually located close to the dso (Ruiz-Masó et al., 2015). During replication, which starts from DSO (with qacC being replicated last) an intermediate stage exists when the single-strand positive strand is covalently bound to Rep at the nick site, to be recircularized when Rep is released. This circular ssDNA is completed to dsDNA starting at the SSO hairpin, again with qacC being replicated last, as shown in Figure 7. If, however, a new nick is introduced in DSO before the minus strand replication round is completely finished, a nicked DNA fragment spanning from the nick site to the SSO, with qacC in between, would be formed. This could be incorporated in an acceptor plasmid by homologous recombination, making use of host recombination proteins. Untimely initiation of replication would normally be prevented when SSO and DSO are close together, but it is quite possible that the presence of a qac gene in between these elements upsets the timing of events.

\section{QacC Is Not the Only Transferable Gene Employing SSO and DSO Sequences}

In search of support for our model, we looked for other genes located between SSO and DSO of RC-plasmids. In species other than Staphylococcus this is quite common for rep or mob genes, and a variety of other genes can be found to separate the two replication elements (e.g., Alonso et al., 1988; Andrup et al., 2003; Alegre et al., 2005; Wu et al., 2007; Zhang et al., 2010, 2011). None of these genes in RC-plasmids from genera other than Staphylococcus share significantly higher homology compared to the rest of their plasmid background, thus there is no indication of gene transfer. Whether other genes on RC-plasmids from genera other than Staphylococcus use this mode of mobilization is currently not known.

However, a second example exists of a gene likely to be transferred by the same mechanism. The gene $\ln u A$, resulting in lincosamide resistance, is located in between SSO and DSO on RC-plasmids of Staphylococcus species, and again it is stronger conserved at the nt level than the sequences outside the proposed mobile locus. As was observed for $q a c C$, the gene can be present on long conjugative plasmids as well as RC-plasmids. In the latter case, the only other gene usually present is rep2. It has been observed before that $\ln u A$ in such plasmids is more strongly conserved than rep (Lüthje et al., 2007). Comparison of nine RC-plasmids by multiple nt alignment identified the segment containing DSO, $\ln u A$ and SSO (694 bp) as $92.7 \%$ conserved, compared to $77 \%$ for the rep gene (coding sequences only) or $74.4 \%$ for the plasmid excluding the DSO-lnuA-SSO segment (ignoring a weakly conserved region). The conserved flank upstream of $\ln u A$ is $78 \mathrm{nt}$ long and the downstream flank is for $160 \mathrm{nt}$ conserved, though the number of direct repeats

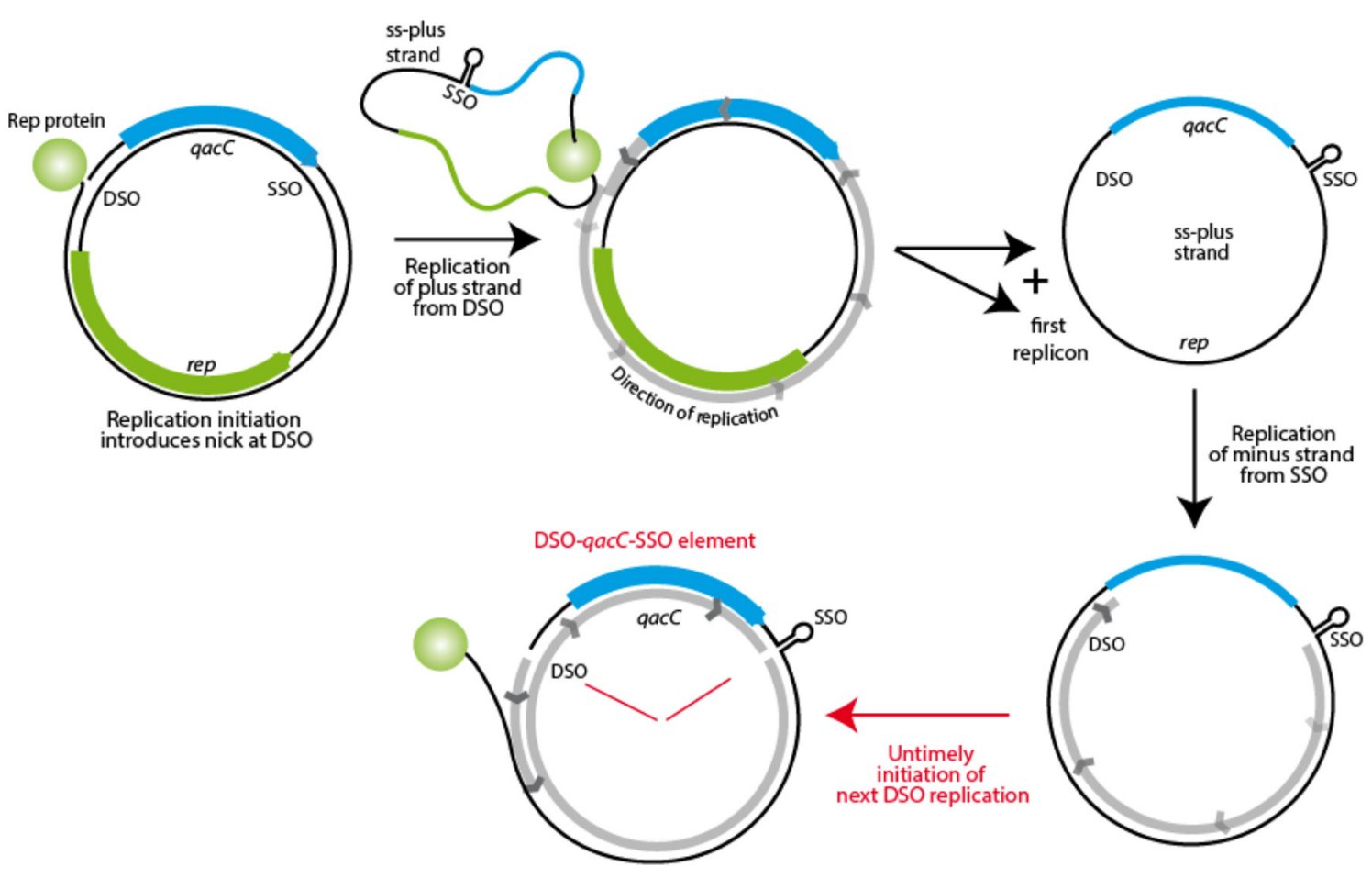

FIGURE 7 | Model for mobility of the qac $\boldsymbol{C}$ locus. The first step in RC-replication is production of the plus strand, resulting in the template plus strand as an ssDNA intermediate. This is subsequently replicated from the SSO, but when a new nick is introduced in this DNA before replication is completed, a fragment containing qacC, flanked by nicked DSO and SSO is the result. This DSO-qacC-SSO element could then be incorporated into an alternative plasmid. 
immediately downstream of $\ln u A$ varies. The circular homology plot is available as Figure S2.

\section{DISCUSSION}

The data presented here suggest a recent spread of $q a c C$ within and between Type I and Type II RC-plasmids. For the DSO-qacC-SSO element to end up in a new plasmid, an acceptor plasmid would probably have to be present together with the donor plasmid in one cell, or at least within the same bacterial population. This requirement has to obey plasmid incompatibility rules. Possibly, Type II, Rep2-containing plasmids are compatible with Type I plasmids that contain Rep1, an assumption that can be experimentally tested. However, our observations further identified spread of the element between plasmids with the same Rep gene. It has to be assumed that in such a case the transfer required cell-cell contact between different plasmid-bearing bacteria, the details of which have not been investigated.

It may not be a coincidence that both examples of a mobile DSO-gene-SSO element identified contain an antimicrobial resistance gene. Possibly, the recent spread of qacC or $\ln u A$ between Staphylococcus plasmids and strains has been selected for by use of disinfectants and antibiotics, and the availability of multiple Staphylococcus plasmid sequences have enabled this discovery. The accumulation of a few mutations suggests the spread of $q a c G$ is less recent than that of qacC.

Of the other qac genes, qacG but not qacJ is likely to have been mobilized recently, based on its pattern of conservation. It is not clear why evidence is lacking that qacJ has recently spread; possibly, the selection for this gene is weaker or the population bearing qacJ has not recently passed a bottleneck. QacJ plasmids were first described from equine isolates of $S$. aureus, Staphylococcus simulans and Staphylococcus intermedius (Bjorland et al., 2003), though human S. aureus (pS1d, pNA32) and Staphylococcus haemolyticus (p92271) isolates can also contain QacJ plasmids. Currently there are too few QacJ plasmid sequences available to draw conclusions about its possible mobility.

\section{REFERENCES}

Alam, M. M., Ishino, M., and Kobayashi, N. (2003). Analysis of genomic diversity and evolution of the low-level antiseptic resistance gene smr in Staphylococcus aureus. Microb. Drug Resist. 9, S1-S7. doi: 10.1089/107662903322541838

Alegre, M. T., Rodríguez, M. C., and Mesas, J. M. (2005). Nucleotide sequence, structural organization and host range of pRS4, a small cryptic Pediococcus pentosaceus plasmid that contains two cassettes commonly found in other lactic acid bacteria. FEMS Microbiol. Lett. 250, 151-156. doi: 10.1016/j.femsle.2005.07.003

Alonso, J. C., Leonhardt, H., and Stiege, C. A. (1988). Functional analysis of the leading strand replication origin of plasmid pUB110 in Bacillus subtilis. Nucleic Acids Res. 16, 9127-9145. doi: 10.1093/nar/16.19.9127

Andrup, L., Jensen, G. B., Wilcks, A., Smidt, L., Hoflack, L., and Mahillon, J. (2003). The patchwork nature of rolling-circle plasmids: comparison of six plasmids from two distinct Bacillus thuringiensis serotypes. Plasmid 49, 205-232. doi: 10.1016/S0147-619X(03)00015-5
Although no experimental evidence has been obtained to support our model of gene mobility, for which a location between the DSO and SSO is crucial, we make a strong case for recent spread of qacC between various Staphylococcus RCplasmids, based on its nearly complete conservation in variable plasmid backgrounds. The hypothesis can be tested by use of a constructed larger sized Type I plasmid with a resistance gene A located between SSO and DSO, and a smaller compatible Type II plasmid with an alternative resistance gene B located at the same position. After double transformation of both plasmids into $S$. aureus under double selection, plasmid DNA could be isolated from the population and physically separated by size. By retransformation of the large plasmid population under selection for resistance gene $B$, a minor population of recombined plasmid DNA might be identified. It is expected that a transfer of gene $B$ does not occur at the same rate in case it is not located in between DSO and SSO.

The discovery of an independent second gene, $\ln u A$, whose higher level of conservation between different plasmids compared to rep is again bordered by DSO and SSO, strengthens the proposed model. This is the first evidence of mobile genes that can be transferred between plasmids without the aid of insertion sequences or transposases. We propose to call this type of mobile DNA an "DSO-gene-SSO" element.

\section{AUTHOR CONTRIBUTIONS}

TW collected the plasmid sequences and performed the alignments. DU provided analysis tools for the initial screens and advised on the presentation of data. HI assisted in the selection of data to be shown and in data presentation and description. All authors contributed to writing of the manuscript.

\section{SUPPLEMENTARY MATERIAL}

The Supplementary Material for this article can be found online at: http://journal.frontiersin.org/article/10.3389/fmicb. 2016.01528

Bikard, D., Loot, C., Baharoglu, Z., and Mazel, D. (2010). Folded DNA in action: hairpin formation and biological functions in prokaryotes. Microbiol. Mol. Biol. Rev. 74, 570-588. doi: 10.1128/MMBR.00026-10

Bjorland, J., Bratlie, M. S., and Steinum, T. (2007). The $s m r$ gene resides on a novel plasmid pSP187 identified in a Staphylococcus pasteuri isolate recovered from unpasteurized milk. Plasmid 57, 145-155. doi: 10.1016/j.plasmid.2006.08.004

Bjorland, J., Steinum, T., Sunde, M., Waage, S., and Heir, E. (2003). Novel plasmid-borne gene qacJ mediates resistance to quaternary ammonium compounds in equine Staphylococcus aureus, Staphylococcus simulans, and Staphylococcus intermedius. Antimicrob. Agents Chemother. 47, 3046-3052. doi: 10.1128/AAC.47.10.3046-3052.2003

Bjorland, J., Steinum, T., Sunde, M., Waage, S., Sviland, S., Oppegaard, H., et al. (2006). Deletion of pT181-like sequence in an smr-encoding mosaic plasmid harboured by a persistent bovine Staphylococcus warneri strain. J. Antimicrob. Chemother. 57, 46-51. doi: 10.1093/jac/dki407

Bjorland, J., Sunde, M., and Waage, S. (2001). Plasmid-borne smr gene causes resistance to quaternary ammonium compounds in 
bovine Staphylococcus aureus. J. Clin. Microbiol. 39, 3999-4004. doi: 10.1128/JCM.39.11.3999-4004.2001

Cho, G. S., Huch, M., Mathara, J. M., van Belkum, M. J., and Franz, C. M. (2013). Characterization of pMRI 5.2, a rolling-circle-type plasmid from Lactobacillus plantarum BFE 5092 which harbours two different replication initiation genes. Plasmid 69, 160-171. doi: 10.1016/j.plasmid.2012.11.005

Costa, S. S., Mourato, C., Viveiros, M., Melo-Cristino, J., Amaral, L., and Couto, I. (2013). Description of plasmid pSM52, harbouring the gene for the Smr efflux pump, and its involvement in resistance to biocides in a meticillinresistant Staphylococcus aureus strain. Int. J. Antimicrob. Agents 41, 490-492. doi: 10.1016/j.ijantimicag.2013.01.003

del Solar, G., Giraldo, R., Ruiz-Echevarría, M. J., Espinosa, M., and Díaz-Orejas, R. (1998). Replication and control of circular bacterial plasmids. Microbiol. Mol. Biol. Rev. 62, 434-464.

Gruss, A. D., Ross, H. F., and Novick, R. P. (1987). Functional analysis of a palindromic sequence required for normal replication of several staphylococcal plasmids. Proc. Natl. Acad. Sci. U.S.A. 8, 2165-2169. doi: 10.1073/pnas.84.8.2165

Heir, E., Sundheim, G., and Holck, A. L. (1995). Resistance to quaternary ammonium compounds in Staphylococcus spp. isolated from the food industry and nucleotide sequence of the resistance plasmid pST827. J. Appl. Bacteriol. 79, 149-156. doi: 10.1111/j.1365-2672.1995.tb00928.x

Khan, S. A. (2005). Plasmid rolling-circle replication: highlights of two decades of research. Plasmid 53, 126-136. doi: 10.1016/j.plasmid.2004.12.008

Kramer, M. G., Espinosa, M., Misra, T. K., and Khan, S. A. (1998). Lagging strand replication of rolling-circle plasmids: specific recognition of the $s s o A$ type origins in different gram-positive bacteria. Proc. Natl. Acad. Sci. U.S.A. 95, 10505-10510. doi: 10.1073/pnas.95.18.10505

Leelaporn, A., Firth, N., Paulsen, I. T., Hettiaratchi, A., and Skurray, R. A. (1995). Multidrug resistance plasmid pSK108 from coagulase-negative staphylococci; relationships to Staphylococcus aureus qacC plasmids. Plasmid 34, 62-67. doi: 10.1006/plas.1995.1034

Lüthje, P., von Köckritz-Blickwede, M., and Schwarz, S. (2007). Identification and characterization of nine novel types of small staphylococcal plasmids carrying the lincosamide nucleotidyltransferase gene $\operatorname{lnu}(\mathrm{A})$. J. Antimicrob. Chemother. 59, 600-606. doi: 10.1093/jac/dkm008

Lyon, B. R., and Skurray, R. (1987). Antimicrobial resistance of Staphylococcus aureus: genetic basis. Microbiol. Rev. 51, 88-134.
Pedersen, A. G., Jensen, L. J., Brunak, S., Staerfeldt, H. H., and Ussery, D. W. (2000). A DNA structural atlas for Escherichia coli. J. Mol. Biol. 299, 907-930. doi: 10.1006/jmbi.2000.3787

Ruiz-Masó, J. A., Machón, C., Bordanaba-Ruiseco, L., Espinosa, M., Coll, M., et al. (2015). Plasmid rolling-circle replication. Microbiol. Spectr. 3:PLAS-0035-2014. doi: 10.1128/microbiolspec.PLAS-0035-2014

Seery, L. T., Nolan, N. C., Sharp, P. M., and Devine, K. M. (1993). Comparative analysis of the pC194 group of rolling circle plasmids. Plasmid 30, 185-196. doi: 10.1006/plas.1993.1051

Thomas, C. D., Balson, D. F., and Shaw, W. V. (1990). In vitro studies of the initiation of staphylococcal plasmid replication. Specificity of RepD for its origin (oriD) and characterization of the Rep-ori tyrosyl ester intermediate. J. Biol. Chem. 265, 5519-5530. Erratum in: J. Biol. Chem. 1990;265: 12749.

Wassenaar, T. M., Ussery, D., Nielsen, L. N., and Ingmer, H. (2015). Review and phylogenetic analysis of qac genes that reduce susceptibility to quaternary ammonium compounds in Staphylococcus species. Eur. J. Microbiol. Immunol. (Bp) 5, 44-61. doi: 10.1556/EuJMI-D-14-00038

Wu, E., Jun, L., Yuan, Y., Yan, J., Berry, C., and Yuan, Z. (2007). Characterization of a cryptic plasmid from Bacillus sphaericus strain LP1-G. Plasmid 57, 296-305. doi: 10.1016/j.plasmid.2006.11.003

Zhang, H., Hao, Y., Zhang, D., and Luo, Y. (2011). Characterization of the cryptic plasmid pTXW from Lactobacillus paracasei TXW. Plasmid 65, 1-7. doi: 10.1016/j.plasmid.2010.08.002

Zhang, Z. H., Tian, W., Liu, D. Y., Liu, Y. C., Shen, Q. R., and Shen, B. (2010). Characterization of a cryptic plasmid pPZZ84 from Bacillus pumilus. Plasmid 64, 200-203. doi: 10.1016/j.plasmid.2010.06.006

Conflict of Interest Statement: The authors declare that the research was conducted in the absence of any commercial or financial relationships that could be construed as a potential conflict of interest.

Copyright (C) 2016 Wassenaar, Ussery and Ingmer. This is an open-access article distributed under the terms of the Creative Commons Attribution License (CC BY). The use, distribution or reproduction in other forums is permitted, provided the original author(s) or licensor are credited and that the original publication in this journal is cited, in accordance with accepted academic practice. No use, distribution or reproduction is permitted which does not comply with these terms. 